\title{
LAUER, Gerhard, UNGER, Thorsten, Das Erdbeben von Lissabon und der Katastrophendiskurs im 18. Jahrhundert
}

\section{François Walter}

\section{(2) OpenEdition}

\section{Journals}

Édition électronique

URL : http://journals.openedition.org/ifha/1839

DOI : $10.4000 /$ ifha. 1839

ISSN : 2198-8943

\section{Éditeur}

IFRA - Institut franco-allemand (sciences historiques et sociales)

Référence électronique

François Walter, «LAUER, Gerhard, UNGER, Thorsten, Das Erdbeben von Lissabon und der Katastrophendiskurs im 18. Jahrhundert », Revue de I'IFHA [En ligne], Date de recension, mis en ligne le 01 janvier 2009, consulté le 22 septembre 2020. URL : http://journals.openedition.org/ifha/1839 ; DOI : https://doi.org/10.4000/ifha.1839

Ce document a été généré automatiquement le 22 septembre 2020.

(C)IFHA 


\title{
LAUER, Gerhard, UNGER, Thorsten, Das Erdbeben von Lissabon und der Katastrophendiskurs im 18. Jahrhundert
}

\author{
François Walter
}

Publié sous la responsabilité de deux professeurs de littérature allemande à l'Université de Göttingen, ce volume reprend les communications d'un colloque de 2005 qui avait réuni, à l'occasion du 250e anniversaire du tremblement de terre de Lisbonne, des géologues, des théologiens, des philosophes, des historiens des sciences, des historiens d'art et des littéraires. Ce fut probablement l'une des plus importantes manifestations organisées à cette occasion. L'excellence des textes ici rassemblés démontre encore une fois, si besoin était, le niveau d'expertise que détiennent les chercheurs allemands dans le champ de l'histoire des catastrophes, sans commune mesure avec ce qu'on a pu entendre dans d'autres colloques du genre notamment en France. En rendre compte suppose soit de passer en revue chacune des 35 communications qui, toutes, mériteraient une mention, soit de donner globalement un aperçu des avancées principales de chacune des grandes thématiques. C'est cette deuxième variante que nous avons retenue. Précisons d'emblée que l'ensemble de la réflexion est toujours menée en phase avec les préoccupations d'aujourd'hui. Ce sont non seulement les modes de perception des événements en leur temps qui sont restitués, mais également les résonances à long terme jusqu'à nos jours d'un tremblement de terre dont on a fait souvent l'une des césures majeures de la modernité. Ce volume est exemplaire dans son objectif de greffer la recherche historique sur une perspective pragmatique de meilleure compréhension des attitudes actuelles face aux catastrophes.

2 La première partie regroupe les communications qui se sont intéressées au mode de médiatisation de l'événement de 1755. Ce sont en effet les médias (avant tout les gazettes) qui ont transformé le tremblement de terre en événement, ce qui ne va pas forcément de soi (le séisme de 1683 en Sicile a fait nettement plus de victimes mais n'a 
pas eu la même audience). La littérature (Goethe par exemple) et la poésie participent également au processus tout comme les arts (surtout la gravure) et même la musique, comme par exemple la cantate de Telemann intitulée Die Donnerode. Lisbonne donne aussi une impulsion à la recherche scientifique. Celle-ci s'efforce de comprendre ce qui s'est passé et contribue donc également au débat public (avec des notions apparues pour la première fois comme l'idée de l'épicentre et de la diffusion des ondes sismiques).

3 Un deuxième groupe de communications aborde une question centrale sur laquelle les propositions sont particulièrement novatrices. En effet, l'une des lignes interprétatives centrales du recueil est de montrer comment, au XVIIIe s., fonctionnent de manière complémentaire deux processus d'attribution de sens : l'un rationaliste, attentif à l'aspect naturel du tremblement de terre, l'autre théologique dans la ligne de l'explication rétributive. Contrairement à ce qu'on a voulu trop longtemps faire accroire, les références religieuses demeurent totalement pertinentes malgré les avancées scientifiques. Les actes du colloque de 2005 comportent plusieurs textes qui apportent des éléments décisifs à un débat que l'on pensait à tort dépassé. En particulier, les auteurs proposent de réévaluer la coupure de 1755 qui ne marque pas unilatéralement la fin de l'optimisme, mais permet des recompositions majeures du côté de la philosophie de l'histoire. Ainsi la position emblématique de Voltaire, dans son récit célèbre (Candide en 1759) et un poème qui ne l'est pas moins, doit être relativisée. Plus caractéristiques de l'esprit des Lumières semblent être les positions de Kant ou même du marquis de Pombal, ministre portugais en charge de la reconstruction de la ville détruite. Ce qui les intéresse, ce n'est plus vraiment la question du bien et du mal que pose sans aucun doute la mort dans la catastrophe de milliers d'innocents, mais les limites de la capacité de l'homme à modifier le monde et à le comprendre. La rationalité consiste à tenter de mesurer les limites de l'agir humain. Lisbonne met en question la confiance que l'homme avait dans son propre pouvoir et le rend désormais plus réceptif au discours théologique. Paradoxalement, la rationalité sceptique n'est pas incompatible avec une théologie plus optimiste. L'un des articles contextualise parfaitement un élément très significatif dans ce débat, à savoir la redécouverte du livre biblique de Job au milieu du siècle.

La troisième partie examine le phénomène majeur de l'esthétisation de la catastrophe. Testée par comparaison avec des sources antiques, à travers la problématique des genres et par la réception de 1755 dans des œuvres littéraires tardives, l'hypothèse de la relecture entraîne à nuancer encore une fois la césure supposée que représenterait Lisbonne. Une quatrième partie décrypte la sémantique politique liée à l'événement. Les auteurs constatent que les nouvelles de la guerre de Sept Ans éclipsent rapidement celles du tremblement de terre. Les effets de Lisbonne se marquent dans le transfert des métaphores géologiques et sismiques vers le champ de la politique. La référence emblématique reste sans doute la petite phrase de Friedrich Schlegel : " Man kann die Französische Revolution als das größte und merkwürdigste Phänomen der Staatengeschichte betrachten, als ein fast universelles Erdbeben, eine unermeßliche Überschwemmung in der politischen Welt. » (" On peut considérer la Révolution française comme le phénomène le plus important et le plus remarquable de l'histoire des États, comme un séisme presque universel, une inondation incommensurable dans le monde politique. »). D'un côté, le transfert du discours catastrophiste pour dire une 
situation politique, de l'autre une instrumentalisation de la situation pour justifier des entreprises de normalisation, telle celle que mène au Portugal le marquis de Pombal.

5 La dernière partie du recueil reprend les communications qui traitent de la perception des risques en économie et dans le champ assuranciel. On apprend que la diffusion d'une conception $\mathrm{du}$ risque comme une probabilité dont l'actualisation est potentiellement calculable a été nettement accélérée par le tremblement de terre lusitanien. On nous rappelle à ce propos les effets évidents sur le développement des assurances et les perfectionnements dans ce que l'on appelle aujourd'hui le management des catastrophes, par exemple à travers de nouvelles normes de construction (techniques qui assurent une certaine élasticité aux bâtiments) et des prescriptions de protection contre les incendies (réglementation de la largeur des rues). Extrêmement originale aussi, la réflexion qui constate comment les mentalités sont prédisposées à accueillir les nouvelles théories de l'évolution, dans la mesure où il est admis que la catastrophe a des effets sur la sélection et l'adaptation des humains à une situation extrême.

6 Plus de 600 pages de qualité pour redéfinir ce que les éditeurs appellent un « moment iconographique "visualisé par la presse, les essais philosophiques, les arts et le discours scientifique : une manière pertinente de montrer l'à-propos de la réflexion historique dans l'analyse des incertitudes du présent.

François WALTER (Université de Genève) 